Published in final edited form as:

Curr Diab Rep. 2005 June ; 5(3): 177-183.

\title{
Mitochondrial Dysfunction and Type 2 Diabetes
}

\author{
Rebecca Parish, MD and Kitt Falk Petersen, MD* \\ Kitt Falk Petersen: kitt.petersen@yale.edu \\ * Department of Internal Medicine, Yale University School of Medicine, 333 Cedar Street, PO Box \\ 208020, New Haven, CT 06520-8020, USA
}

\begin{abstract}
Insulin resistance plays a major role in the pathogenesis of the metabolic syndrome and type 2 diabetes, and yet the mechanisms responsible for it remain poorly understood. Magnetic resonance spectroscopy studies in humans suggest that a defect in insulin-stimulated glucose transport in skeletal muscle is the primary metabolic abnormality in insulin-resistant patients with type 2 diabetes. Fatty acids appear to cause this defect in glucose transport by inhibiting insulinstimulated tyrosine phosphorylation of insulin receptor substrate-1 (IRS-1) and IRS-1-associated phosphatidylinositol 3-kinase activity. A number of different metabolic abnormalities may increase intramyocellular and intrahepatic fatty acid metabolites; these include increased fat delivery to muscle and liver as a consequence of either excess energy intake or defects in adipocyte fat metabolism, and acquired or inherited defects in mitochondrial fatty acid oxidation. Understanding the molecular and biochemical defects responsible for insulin resistance is beginning to unveil novel therapeutic targets for the treatment of the metabolic syndrome and type 2 diabetes.
\end{abstract}

\section{Introduction}

Type 2 diabetes is one of the fastest growing public health problems in the increasingly obese western society [1]. By the year 2020, more than 250 million people will be afflicted worldwide, resulting in a substantial financial burden, with more than $\$ 100$ billion spent annually in the United States alone. Cross-sectional studies have demonstrated the presence of insulin resistance in virtually all patients with type 2 diabetes, and prospective studies have demonstrated the presence of insulin resistance one to two decades prior to the onset of the disease [2-4]. Insulin resistance in the offspring of parents with type 2 diabetes has been shown to be the best predictor for the later development of the disease [5], and perturbations that reduce insulin resistance may prevent the development of diabetes [6]. Therefore, it is important to understand the pathogenic mechanisms of insulin resistance in order to identify novel targets for primary and secondary prevention. In this article, we briefly review some of the recent advances in our understanding of insulin resistance in humans; the interventions that are effective in reversing insulin resistance and possibly the progression to type 2 diabetes; and the mechanisms of diabetes therapy, both pharmacologic and nonpharmacologic, that improve insulin sensitivity, when progression to diabetes does occur.

\section{Cellular Mechanisms of Muscle Insulin Resistance}

Our group has focused on the use of magnetic resonance spectroscopy (MRS) to examine intracellular metabolism in humans. MRS is noninvasive and involves no ionizing radiation. It can be used to directly measure concentrations of intracellular metabolites, such as glucose, glucose-6-phosphate (G-6-P), triglyceride, and glycogen, which typically cannot be measured accurately or in a repeated manner with conventional biopsy techniques. 
Using ${ }^{13} \mathrm{C}$ MRS, we noninvasively measured rates of muscle glycogen synthesis in patients with type 2 diabetes under steady-state plasma concentrations of insulin and glucose that mimic postprandial conditions. We found that muscle glycogen synthesis was approximately $50 \%$ lower than in normal volunteers. In both patients with type 2 diabetes and the normal volunteers, muscle glycogen synthesis accounted for virtually all of the whole-body insulinstimulated glucose uptake in both normal and diabetic subjects [7]. Thus, these studies demonstrate that under hyperglycemic, hyperinsulinemic conditions, muscle glycogen synthesis is the major pathway for glucose metabolism in both normal and diabetic individuals, and that defective muscle glycogen synthesis plays a major role in causing insulin resistance in patients with type 2 diabetes.

In order to determine the rate-controlling steps for this defect, we combined ${ }^{13} \mathrm{C}$ and ${ }^{31} \mathrm{P}$ MRS studies to simultaneously measure rates of muscle glycogen synthesis along with concentrations of G-6-P in patients with type 2 diabetes and age-weight-matched control subjects [8]. Intracellular G-6-P is an intermediary metabolite between glucose transport/ phosphorylation and glycogen synthesis, and the intracellular concentration of G-6-P will respond to the relative activities of these two steps. G-6-P cannot be accurately measured in human muscle biopsies because its concentration increases due to the rapid hydrolysis of glycogen to G-6-P during the muscle biopsy procedure. Decreased activity of glycogen synthase in diabetes would lead to an increase in G-6-P concentrations relative to that of normal individuals. We found that in the type 2 diabetic patients the insulin-stimulated changes in G-6-P were significantly blunted, suggesting either decreased glucose transport activity or decreased glucose phosphorylation activity (via hexokinase II) as the cause of their muscle insulin resistance.

Because this defect could be caused by glucose toxicity and other factors, we studied young, healthy, lean, insulin-resistant (IR) offspring of parents with type 2 diabetes using a similar study protocol. Young, lean, healthy offspring of parents with type 2 diabetes have an approximate $40 \%$ risk of developing diabetes later in life, and insulin resistance is the best predictor for whether this happens.

We found that these IR offspring, like their parents with type 2 diabetes, had a 50\% reduction in the rate of insulin-stimulated whole-body glucose metabolism, which could be accounted for by a decrease in the rate of muscle glycogen synthesis [9]. This reduction in insulin-stimulated muscle glycogen synthesis was associated with a blunted increment of insulin-stimulated intramuscular G-6-P concentration. Taken together, these data suggest that defects in insulin-stimulated muscle glucose transport and phosphorylation activity are very early events in the pathogenesis of type 2 diabetes.

Finally, to determine whether glucose transport or phosphorylation are rate controlling in patients with type 2 diabetes, we developed a ${ }^{13} \mathrm{C}$ MRS technique to noninvasively assess intracellular free glucose concentrations in muscle [10]. Intracellular glucose is an intermediary metabolite between glucose transport and glucose phosphorylation, and its concentration reflects the relative activities of these two steps in muscle glucose metabolism.

We found in patients with type 2 diabetes that the intracellular glucose concentrations were far lower than the concentrations expected if hexokinase II was the primary rate-controlling enzyme for glycogen synthesis, strongly suggesting that defective insulin-stimulated glucose transport activity is the major factor responsible for insulin resistance in patients with type 2 diabetes. 


\section{Fat-induced Muscle Insulin Resistance}

Plasma free fatty acid concentrations are increased in many insulin-resistant states, including obesity and type 2 diabetes mellitus [11]. In a cross-sectional study of healthy, young, lean offspring of type 2 diabetic patients, we found an inverse relationship between intramyocellular lipid (IMCL) content as measured by ${ }^{1} \mathrm{H}$ MRS and insulin sensitivity, consistent with the hypothesis that altered fatty acid metabolism contributes to insulin resistance in patients with type 2 diabetes [12].

In a recent series of studies by our group, we examined this question of fat-induced insulin resistance in humans [13]. Using ${ }^{13} \mathrm{C}$ and ${ }^{31} \mathrm{P}$ MRS to measure skeletal muscle glycogen and G-6-P concentrations in healthy subjects under conditions of euglycemia and hyperinsulinemia, we increased levels of plasma free fatty acids during an infusion of a lipid and heparin emulsion. Five hours of maintaining high levels of plasma fatty acid concentrations resulted in a 50\% reduction in insulin-stimulated rates of muscle glycogen synthesis and whole-body glucose oxidation compared with the control study (in the absence of any exogenous fatty acids). Most importantly, the 50\% reduction in muscle glycogen synthesis was preceded by a decrement in intramuscular G-6-P concentrations. Our findings suggest that increases in plasma fatty acid concentrations initially induce insulin resistance by inhibiting glucose transport or phosphorylation activity, and that the reduction in muscle glycogen synthesis and glucose oxidation follows. The reduction in insulin-activated glucose transport and phosphorylation activity in normal subjects maintained at high plasma fatty acid levels is similar to the reduction seen in obese individuals [14], patients with type 2 diabetes [8], and lean, normoglycemic IR offspring of type 2 diabetic individuals [9]. Thus, it appears that accumulation of intramuscular fatty acid metabolites play an important role in the pathogenesis of insulin resistance in obese patients and patients with type 2 diabetes.

To further distinguish between possible effects of fatty acids on glucose transport activity and hexokinase II activity, we measured intracellular concentrations of glucose in muscle using ${ }^{13} \mathrm{C}$ MRS [15]. We found that elevated plasma fatty acid concentrations significantly reduced intracellular glucose concentrations, implying that the rate-controlling step for fatty acid-induced insulin resistance in humans is glucose transport.

This reduction in glucose transport activity could be caused by direct effects of the fatty acids on the GLUT4 transporter, such as alterations in the trafficking, budding, fusion, or activity of GLUT4, or the fatty acids could induce alterations in upstream insulin signaling events, resulting in decreased GLUT4 translocation to the plasma membrane. We examined the effects of insulin stimulation on insulin receptor substrate-1 (IRS-1)-associated phosphatidylinositol 3-kinase (PI3-kinase) activity in muscle biopsies using the same lipid and heparin emulsion infusion protocol described earlier. We found that high plasma free fatty acid concentrations abolished the fourfold increase in insulin-stimulated, IRS-1associated PI3-kinase activity observed in the glycerol-control studies [15].

The effects of intracellular fatty acids (or some fatty acid metabolite) may be a direct reduction of the insulin-stimulated PI3-kinase activity or it may be secondary to alterations in upstream insulin signaling events. Consistent with an indirect effect, we found in rats that the lipid and heparin infusion raising plasma free fatty acids resulted in a reduction of insulin-stimulated IRS- 1 tyrosine phosphorylation and activation of protein kinase $\mathrm{C} \theta$ serine kinase, which can be activated by fatty acid metabolites such as diacylglycerol [16]. Thus, one hypothesis to account for the effects of fatty acids to cause insulin resistance in muscle cells may be that increasing intracellular fatty acid metabolites, such as diacylglycerol or fatty acyl coenzyme As (CoAs), leads to phosphorylation of serine and threonine sites on IRS- 1 by activation of a serine and threonine kinase cascade involving protein kinase $C \theta^{19}$ 
and possibly other serine kinases, such as IkB kinase- $\beta$ or IKK- $\beta$ [17-22] and c-Jun aminoterminal kinases [23]. Serine-phosphorylated forms of these proteins fail to associate with and activate PI3-kinase, resulting in decreased activation of glucose transport and other downstream-associated events. If this hypothesis is correct, any perturbation that results in accumulation of intracellular fatty acyl CoAs or other fatty acid metabolites in muscle and liver (ie, excess caloric intake, alterations in adipocyte fatty acid metabolism, or through decreased mitochondrial fatty acid oxidation) may be expected to induce insulin resistance in these organs [24]. All of these possibilities appear to occur in humans.

\section{Increased Energy Intake, Obesity, and Insulin Resistance}

It is well established that too much body fat is strongly associated with insulin resistance and the most common cause of insulin resistance occurs when energy intake exceeds energy expenditure, leading to obesity [14]. Under these conditions, increased delivery of dietary fat or delivery of endogenous fat from increased hepatic lipogenesis results in accumulation of fatty acid metabolites in liver and muscle, which subsequently causes insulin resistance through the mechanisms described earlier. This would mean that obesity invariably leads to insulin resistance; however, it is clear that not all obese individuals are IR. Several studies have described that obesity, where the fat is stored centrally (android or apple-shaped distribution), is typically associated with insulin resistance [24,25]. In contrast, when obesity is associated with fat stored around the hips (gynoid or pear-shaped distribution), insulin sensitivity remains normal. Although it is widely believed that adipocyte-derived cytokines that are released by the visceral fat, such as tumor necrosis factor- $\alpha$ [26], interleukin-6 [27], adiponectin [28], and visfatin [29,30], may be responsible for the insulin resistance in android-shaped individuals, an alternative explanation is that visceral fat cells do not store fat as effectively as peripheral fat cells and that these individuals have redistributed their fat to intracellular sites in liver and muscle cells, resulting in insulin resistance in these tissues. In contrast, individuals with a gynoid distribution of fat (sometimes referred to as the "fit and fat" individual) store excess fat in peripheral adipocytes, which store fat more effectively and keep fat out of muscle and liver.

\section{Defects in Adipocyte Fatty Acid Metabolism and Insulin Resistance}

Evidence for defects in adipocyte metabolism causing insulin resistance comes from recent studies in both transgenic mouse models of lipodystrophy and patients with severe lipodystrophy. Transgenic mice expressing A-ZIP/F-1 are almost totally devoid of fat because the A-ZIP/F-1 protein blocks the function of several classes of transcription factors [31]. Interestingly, these fatless mice also manifest severe liver and muscle insulin resistance and they develop diabetes [32,33]. Furthermore, they have a twofold increase in fatty acyl CoA content in muscle and liver, and defects in insulin activation of IRS-1 and IRS-2associated PI3-kinase in both of these tissues. Transplantation of fat tissue from normal littermates into these fatless mice normalized fatty acyl CoA content in muscle and liver and normalized insulin signaling and action in liver and muscle [33].

Similar results have been seen in patients with severe lipodystrophy. These patients were found to have severe insulin resistance in both liver and muscle, which was associated with severe hepatic steatosis but surprisingly no increment of IMCL despite severe muscle insulin resistance [34•]. These data are consistent with recent studies that have demonstrated that intramyocellular triglyceride is not the trigger in mediating muscle insulin resistance, but more likely a marker for some other intracellular fatty acid metabolite [21]. Prior to leptin treatment, there was no rational treatment for severe, generalized lipodystrophy.

Replacement leptin therapy for 3 to 8 months reversed both hepatic and muscle insulin resistance, and these changes were associated with a reduction in both intrahepatic 
triglyceride and IMCL content [34•]. In these patients, the complete lack of fat cells combined with hyperphagia was causing excess fat storage in muscle and liver.

These findings offer further evidence in support of the hypothesis that insulin resistance develops in obesity, type 2 diabetes, and lipodystrophy because of alterations in the partitioning of fat between the adipocyte and muscle and liver. This, in turn, leads to the accumulation of intracellular fatty acid metabolites ( $\mathrm{eg}$, fatty acyl CoAs, diacylglycerol) in muscle and liver, which then leads to activation of a serine kinase cascade, leading to defects in insulin signaling and insulin action in these tissues.

\section{Defects in Mitochondrial Function and Insulin Resistance}

It may also be expected that any alteration in the ability of muscle and liver to metabolize fatty acids, such as inherited or acquired defects in mitochondrial function [35], would lead to intracellular accumulation of fatty acid metabolites and subsequent defects in insulin signaling and action [31]. A recent study by our group using ${ }^{13} \mathrm{C}$ and ${ }^{31} \mathrm{P}$ MRS techniques to assess mitochondrial oxidative-phosphorylation activity demonstrated that insulin resistance in the elderly could be attributed to increased IMCL and intrahepatic lipid content, which in turn was linked to a reduction in mitochondrial oxidative-phosphorylation activity [36•]. The reduction in mitochondrial function and lipid accumulation in muscle and liver can likely be ascribed to an age-associated reduction in mitochondrial content due to accumulated mutations in mitochondrial DNA, which have been described to occur with aging [37].

In more recent studies, using the same ${ }^{31} \mathrm{P}$ MRS techniques to assess rates of ATP synthesis in skeletal muscle, we found similar reductions in mitochondrial activity associated with an increase in IMCL content in young, lean, IR offspring [38•]. Taken together, these data suggest that alterations in nuclear-encoded genes that regulate mitochondrial biogenesis, such as PGC-1- $\alpha$ [39,40], AMP kinase [41], and CAM IV [42], may form the genetic basis for inheritance of type 2 diabetes. This hypothesis is supported by recent microarray data in patients with type 2 diabetes and their first-degree relatives [43].

\section{Exercise and Insulin Resistance}

Exercise increases energy expenditure and insulin sensitivity, so we examined whether chronic exercise training could reverse the defect in glucose transport and phosphorylation activity in IR offspring $[44,45]$. Over a period of 6 weeks a cohort of young, healthy, lean IR offspring exercised four times $40 \mathrm{~min} / \mathrm{wk}$ on a Stairmaster (Nautilus, Tulsa, OK) at $65 \%$ of their maximum oxygen consumption. Following this exercise regimen, insulin sensitivity and insulin-stimulated muscle glycogen synthesis normalized in the IR offspring, and this could be attributed to correction of their defects in muscle glucose transport and phosphorylation activity. The results of this study strongly suggest that aerobic exercise may be useful in reversing insulin resistance in these prediabetic individuals and that it, thus, may prevent the development of type 2 diabetes. This hypothesis is supported by the recent data from the Diabetes Prevention Program study, which demonstrated that the combination of diet and exercise is effective in decreasing the incidence of type 2 diabetes in patients with impaired glucose tolerance [46].

\section{Moderate Weight Reduction in Patients with Type 2 Diabetes}

Henry et al. [47] showed that a relatively modest weight reduction in obese, poorly controlled type 2 diabetic subjects can markedly reduce plasma glucose concentrations, although the mechanism responsible for this phenomenon was poorly understood. Based on our studies in lipodystrophic patients, where the leptin-induced decrement in hepatic lipid content was associated with reversal of hepatic insulin resistance, we hypothesized that a 
relatively small pool of intrahepatic lipid may be responsible for the hepatic insulin resistance and fasting hyperglycemia in these individuals [34•]. In order to examine this hypothesis, we measured rates of hepatic glucose production and hepatic insulin sensitivity along with intrahepatic and IMCL contents weekly by ${ }^{1} \mathrm{H}$ MRS in eight obese type 2 diabetic subjects in moderate to poor glycemic control before, during, and after 7 weeks of weight loss $(1200 \mathrm{kcal} / \mathrm{d})$ [48]. The diabetic subjects had increased rates of fasting hepatic glucose production, which was associated with severe hepatic and peripheral insulin resistance. These changes were associated with severe hepatic steatosis and marked increases in IMCL content. A weight loss of only approximately $8 \mathrm{~kg}$ resulted in normalization of fasting plasma glucose concentrations and rates of fasting hepatic glucose production. In addition, hepatic insulin sensitivity normalized, which could be attributed to an $80 \%$ reduction in hepatic lipid content. In contrast, there was no improvement in peripheral insulin resistance or change in their IMCL content.

In a subgroup of patients, rates of net hepatic glycogenolysis and gluconeogenesis were assessed before and after weight loss by ${ }^{13} \mathrm{C}$ MRS. Prior to the weight reduction, the increased rates of glucose production could be completely accounted for by increased rates of gluconeogenesis. Following weight loss, the reduced rates of glucose production could be entirely attributed to a reduction in the rates of gluconeogenesis. These data support the hypothesis that moderate weight loss normalizes fasting hyperglycemia in poorly controlled type 2 diabetic patients, by mobilizing a relatively small pool of hepatic lipids, which reverses hepatic insulin resistance and normalizes rates of basal glucose production, independent of any changes in insulin-stimulated peripheral glucose metabolism [48].

\section{Pharmacologic Treatment: The Liver}

Increased rates of glucose production are strongly correlated with increased fasting plasma glucose concentrations in patients with type 2 diabetes [49,50]. In a previous study using ${ }^{13} \mathrm{C}$ MRS to assess the contributions of net hepatic glycogenolysis and gluconeogenesis to glucose production in patients with type 2 diabetes, we found that increased gluconeogenesis can account for the increase in rates of glucose production in these patients [51]. In order to examine the mechanism by which metformin lowers glucose production in patients with type 2 diabetes, we studied seven type 2 diabetic patients in poor glycemic control (glycosylated hemoglobin of 13\%) before and after 3 months of metformin therapy (and compared to an age-and weight-matched control group) [52]. Rates of net hepatic glycogenolysis, assessed by ${ }^{13} \mathrm{C}$ MRS, were combined with an infusion of $\left[6,6-{ }^{2} \mathrm{H}_{2}\right]$ glucose to determine rates of glucose production and the contributions to glucose production of gluconeogenesis and glycogenolysis $[53,54]$. Before treatment with metformin, the rate of glucose production was twice as high in the patients with type 2 diabetes and the rate of gluconeogenesis was three times higher compared with the control subjects. Metformin reduced fasting plasma glucose concentrations by $30 \%$, rates of glucose production by $24 \%$, and gluconeogenesis by $30 \%$ to $36 \%$. The study showed that the increase in rates of glucose production in the diabetic patients could be accounted for by an increase in gluconeogenesis and that metformin treatment improved glycemic control and decreased rates of glucose production via a reduction in the rate of gluconeogenesis.

\section{Pharmacologic Treatment: The Muscle}

The hypothesis that several forms of insulin resistance in humans may be caused by accumulation of intracellular fatty acid metabolites (which may activate a serine or threonine cascade causing serine phosphorylation of IRS- 1 at critical sites leading to failure of IRS- 1 to associate with and activate PI3-kinase, and thus decreased activation of glucose transport) may also explain how thiazolidinediones improve insulin sensitivity in muscle and 
liver tissue. By activation of peroxisome proliferator-activated receptor- $\gamma$ receptors in the adipocyte and promotion of adipocyte differentiation, these agents may facilitate a redistribution of fat from liver and muscle into the adipocytes, much as fat transplantation does in fat-deficient lipodystrophic mice [32,33]. This hypothesis is supported by a recent study in which 3 months of rosiglitazone therapy in patients with type 2 diabetes improved muscle insulin responsiveness and was associated with a marked reduction in intrahepatic fat content, a decrease in the IMCL or extracellular lipid content, and increased peripheral adipocyte insulin sensitivity to suppress lipolysis [55•].

\section{Conclusions}

Insulin resistance plays a major role in the pathogenesis of type 2 diabetes. In this review, we discussed some recent MRS studies that potentially shed new light on the pathogenesis of insulin resistance in humans. 1) Insulin resistance in skeletal muscle can mostly be attributed to defects in insulin-stimulated glucose transport activity. 2) Fatty acids cause insulin resistance in human skeletal muscle by directly interfering with insulin-stimulated glucose transport activity. 3) Reduced insulin activation of glucose transport activity can be attributed to an acquired defect in insulin-stimulated IRS-1-associated PI3-kinase activity at the level of IRS-1 tyrosine phosphorylation. This, in turn, can be ascribed to fatty acid activation of a serine kinase cascade, which causes increased serine phosphorylation of IRS-1 at critical sites that interfere and block tyrosine phosphorylation of IRS-1 tyrosine sites that are required to bind and activate PI3-kinase. 4) Any perturbation that leads to an increase in intramyocellular fatty acid metabolite (eg, fatty acyl CoAs, diacylglycerol, and so forth) content, such as acquired ( $e g$, aging) or inherited (eg, potential type 2 diabetes genes) defects in mitochondrial fatty acid oxidation, defects in adipocyte fat metabolism (eg, lipodystrophy) leading to increased fat delivery to liver and muscle or most commonly increased fat delivery due to increased caloric intake, will lead to insulin resistance through this final common pathway. 5) Interventions to decrease the content of intracellular fatty acid metabolites, such as exercise, diet, and targeted pharmacologic treatment, which will decrease the lipid content of insulin-sensitive tissues or redistribute the fat from the muscle and liver to the adipocytes, will improve insulin sensitivity. Finally, mitochondrial dysfunction, be it acquired or inherited, may be an important contributing factor to the development of obesity. Understanding these key cellular mechanisms of insulin resistance should help elucidate new targets for treating or preventing type 2 diabetes.

\section{References and Recommended Reading}

Papers of particular interest, published recently, have been highlighted as:

- Of importance

•- Of major importance

1. Zimmet P, Alberti KG, Shaw J. Global and societal implications of the diabetes epidemic. Nature 2001;414:782-787. [PubMed: 11742409]

2. Lillioja S, Mott DM, Howard BV, et al. Impaired glucose tolerance as a disorder of insulin action. Longitudinal and cross-sectional studies in Pima Indians. N Engl J Med 1988;318:1217-1225. [PubMed: 3283552]

3. Lillioja S, Mott DM, Spraul M, et al. Insulin resistance and insulin secretory dysfunction as precursors of non-insulin-dependent diabetes mellitus. Prospective studies of Pima Indians. N Engl J Med 1993;329:1988-1992. [PubMed: 8247074]

4. DeFronzo RA. Pathogenesis of type 2 (non-insulin dependent) diabetes mellitus: a balanced overview. Diabetologia 1992;35:389-397. [PubMed: 1516769] 
5. Warram JH, Martin BC, Krolewski AS, et al. Slow glucose removal rate and hyperinsulinemia precede the development of type II diabetes in the offspring of diabetic parents. Ann Intern Med 1990;113:909-915. [PubMed: 2240915]

6. Azen SP, Peters RK, Berkowitz K, et al. TRIPOD (TRoglitazone In the Prevention Of Diabetes): a randomized, placebo-controlled trial of troglitazone in women with prior gestational diabetes mellitus. Control Clin Trials 1998;19:217-231. [PubMed: 9551285]

7. Shulman GI, Rothman DL, Jue T, et al. Quantitation of muscle glycogen synthesis in normal subjects and subjects with non-insulin-dependent diabetes by $13 \mathrm{C}$ nuclear magnetic resonance spectroscopy. N Engl J Med 1990;322:223-228. [PubMed: 2403659]

8. Rothman DL, Shulman RG, Shulman GI. 31P nuclear magnetic resonance measurements of muscle glucose-6-phosphate. Evidence for reduced insulin-dependent muscle glucose transport or phosphorylation activity in non-insulin-dependent diabetes mellitus. J Clin Invest 1992;89:10691075. [PubMed: 1556176]

9. Rothman DL, Magnusson I, Cline G, et al. Decreased muscle glucose transport/phosphorylation is an early defect in the pathogenesis of non-insulin-dependent diabetes mellitus. Proc Natl Acad Sci U S A 1995;92:983-987. [PubMed: 7862678]

10. Cline GW, Petersen KF, Krssak M, et al. Impaired glucose transport as a cause of decreased insulin-stimulated muscle glycogen synthesis in type 2 diabetes. N Engl J Med 1999;341:240-246. [PubMed: 10413736]

11. Boden G, Shulman GI. Free fatty acids in obesity and type 2 diabetes: defining their role in the development of insulin resistance and beta-cell dysfunction. Eur J Clin Invest 2002;32(suppl 3): 14-23. [PubMed: 12028371]

12. Krssak M, Petersen KF, Dresner A, et al. Intramyocellular lipid concentrations are correlated with insulin sensitivity in man: a 1H NMR spectroscopy study. Diabetologia 1999;41:113-116. [PubMed: 10027589]

13. Roden M, Price TB, Perseghin G, et al. Mechanism of free fatty acid-induced insulin resistance in humans. J Clin Invest 1996;97:2859-2865. [PubMed: 8675698]

14. Petersen KF, Hendler R, Price T, et al. 13C/31P NMR studies on the mechanism of insulin resistance in obesity. Diabetes 1998;47:381-386. [PubMed: 9519743]

15. Dresner A, Laurent D, Marcucci M, et al. Effects of free fatty acids on glucose transport and IRS-1-associated phosphatidylinositol 3-kinase activity. J Clin Invest 1999;103:253-259. [PubMed: 9916137]

16. Griffin ME, Marcucci MJ, Cline GW, et al. Free fatty acid-induced insulin resistance is associated with activation of protein kinase $\mathrm{C}$ theta and alterations in the insulin signaling cascade. Diabetes 1999;48:1270-1274. [PubMed: 10342815]

17. Yin MJ, Yamamoto Y, Gaynor RB. The anti-inflammatory agents aspirin and salicylate inhibit the activity of I(kappa)B kinase-beta. Nature 1998;396:77-80. [PubMed: 9817203]

18. Yuan M, Konstantopoulos N, Lee J, et al. Reversal of obesity- and diet-induced insulin resistance with salicylates or targeted disruption of Ikkbeta. Science 2001;293:1673-1677. [PubMed: 11533494]

19. Kim JK, Kim YJ, Fillmore JJ, et al. Prevention of fat-induced insulin resistance by salicylate. J Clin Invest 2001;108:437-446. [PubMed: 11489937]

20. Hundal RS, Petersen KF, Mayerson AB, et al. Mechanism by which high-dose aspirin improves glucose metabolism in type 2 diabetes. J Clin Invest 2002;109:1321-1326. [PubMed: 12021247]

21. Yu C, Chen Y, Cline GW, et al. Mechanism by which fatty acids inhibit insulin activation of insulin receptor substrate-1 (IRS-1)-associated phosphatidylinositol 3-kinase activity in muscle. J Biol Chem 2002;277:50230-50236. [PubMed: 12006582]

22. Itani SI, Ruderman NB, Schmieder F, Boden G. Lipid-induced insulin resistance in human muscle is associated with changes in diacylglycerol, protein kinase C, and IkappaB-alpha. Diabetes 2002;51:2005-2011. [PubMed: 12086926]

23. Hotamisligil GS, Peraldi P, Budavari A, et al. IRS-1-mediated inhibition of insulin receptor tyrosine kinase activity in TNF-alpha- and obesity-induced insulin resistance. Science 1996;271:665-668. [PubMed: 8571133] 
24. Bjorntorp P. Abdominal fat distribution and the metabolic syndrome. J Cardiovasc Pharmacol 1992;20(suppl 8):S26-S28. [PubMed: 1283766]

25. Foley JE, Thuillez P, Lillioja S, et al. Insulin sensitivity in adipocytes from subjects with varying degrees of glucose tolerance. Am J Physiol 1986;251:E306-E310. [PubMed: 3529983]

26. Saghizadeh M, Ong JM, Garvey WT, et al. The expression of TNF alpha by human muscle. Relationship to insulin resistance. J Clin Invest 1996;97:1111-1116. [PubMed: 8613535]

27. Beisel WR. Herman Award Lecture, 1995: infection-induced malnutrition-from cholera to cytokines. Am J Clin Nutr 1995;62:813-819. [PubMed: 7572715]

28. Steppan CM, Lazar MA. Resistin and obesity-associated insulin resistance. Trends Endocrinol Metab 2002;13:18-23. [PubMed: 11750858]

29. Fukuhara A, Matsuda M, Nishizawa M, et al. Visfatin: a protein secreted by visceral fat that mimics the effects of insulin. Science 2005;307:426-430. [PubMed: 15604363]

30. Hug C, Lodish HF. Medicine. Visfatin: a new adipokine. Science 2005;307:366-367. [PubMed: 15604359]

31. Moitra J, Mason MM, Olive M, et al. Life without white fat: a transgenic mouse. Genes Dev 1998;12:3168-3181. [PubMed: 9784492]

32. Gavrilova O, Marcus-Samuels B, Graham D, et al. Surgical implantation of adipose tissue reverses diabetes in lipoatrophic mice. J Clin Invest 2000;105:271-278. [PubMed: 10675352]

33. Kim JK, Gavrilova O, Chen Y, et al. Mechanism of insulin resistance in A-ZIP/F-1 fatless mice. J Biol Chem 2000;275:8456-8460. [PubMed: 10722680]

34- Petersen KF, Oral EA, Dufour S, et al. Leptin reverses insulin resistance and hepatic steatosis in patients with severe lipodystrophy. J Clin Invest 2002;109:1345-1350. Showed that patients with generalized lipodystrophy had severe type 2 diabetes, hepatic steatosis, and hepatic insulin resistance in the absence of intra-abdominal fat. During leptin replacement treatment, the hepatic lipid stores were mobilized and this was associated with normalization of hepatic glucose production and suppression by insulin. The study strongly supports the hypothesis that hepatic lipid accumulation causes hepatic insulin resistance. [PubMed: 12021250]

35. Conley KE, Jubrias SA, Esselman PC. Oxidative capacity and ageing in human muscle. J Physiol 2000;526(Pt 1):203-210. [PubMed: 10878112]

36•. Petersen KF, Befroy D, Dufour S, et al. Mitochondrial dysfunction in the elderly: possible role in insulin resistance. Science 2003;300:1140-1142. The insulin clamp and ${ }^{13} \mathrm{C}$ and ${ }^{31} \mathrm{P}$ MRS techniques were used to assess muscle insulin sensitivity and mitochondrial oxidative function in lean, sedentary elderly subjects. The findings support the hypothesis that an age-associated decline in mitochondrial function contributes to insulin resistance in the elderly by an ageassociated accumulation of IMCL. [PubMed: 12750520]

37. Michikawa Y, Mazzucchelli F, Bresolin N, et al. Aging-dependent large accumulation of point mutations in the human mtDNA control region for replication. Science 1999;286:774-779. [PubMed: 10531063]

38- Petersen KF, Dufour S, Befroy D, et al. Impaired mitochondrial activity in the insulin-resistant offspring of patients with type 2 diabetes. N Engl J Med 2004;350:664-671. Illustrated that insulin resistance in lean offspring of parents with type 2 diabetes is associated with dysregulation of intramyocellular fatty acid metabolism, possibly due to an inherited defect in mitochondrial oxidative function. [PubMed: 14960743]

39. Wu Z, Puigserver P, Andersson U, et al. Mechanisms controlling mitochondrial biogenesis and respiration through the thermogenic coactivator PGC-1. Cell 1999;98:115-124. [PubMed: 10412986]

40. Lin J, Wu H, Tarr PT, et al. Transcriptional co-activator PGC-1 alpha drives the formation of slowtwitch muscle fibres. Nature 2002;418:797-801. [PubMed: 12181572]

41. Zong H, Ren JM, Young LH, et al. AMP kinase is required for mitochondrial biogenesis in skeletal muscle in response to chronic energy deprivation. Proc Natl Acad Sci U S A 2002;99:1598315987. [PubMed: 12444247]

42. Wu H, Kanatous SB, Thurmond FA, et al. Regulation of mitochondrial biogenesis in skeletal muscle by CaMK. Science 2002;296:349-352. [PubMed: 11951046] 
43. Patti ME, Butte AJ, Crunkhorn S, et al. Coordinated reduction of genes of oxidative metabolism in humans with insulin resistance and diabetes: Potential role of PGC1 and NRF1. Proc Natl Acad Sci U S A 2003;100:8466-8471. [PubMed: 12832613]

44. Mikines KJ, Sonne B, Farrell PA, et al. Effect of physical exercise on sensitivity and responsiveness to insulin in humans. Am J Physiol 1988;254:E248-E259. [PubMed: 3126668]

45. Perseghin G, Price TB, Petersen KF, et al. Increased glucose transport-phosphorylation and muscle glycogen synthesis after exercise training in insulin-resistant subjects. $\mathrm{N}$ Engl J Med 1996;335:1357-1362. [PubMed: 8857019]

46. Knowler WC, Barrett-Connor E, Fowler SE, et al. Reduction in the incidence of type 2 diabetes with lifestyle intervention or metformin. N Engl J Med 2002;346:393-403. [PubMed: 11832527]

47. Henry RR, Scheaffer L, Olefsky JM. Glycemic effects of intensive caloric restriction and isocaloric refeeding in noninsulin-dependent diabetes mellitus. J Clin Endocrinol Metab 1985;61:917-925. [PubMed: 4044780]

48. Petersen KF, Dufour S, Befroy D, et al. Reversal of nonalcoholic hepatic steatosis, hepatic insulin resistance and hyperglycemia by moderate weight reduction in patients with type 2 diabetes mellitus. Diabetes 2005;54:603-608. [PubMed: 15734833]

49. Jeng CY, Sheu WH, Fuh MM, et al. Relationship between hepatic glucose production and fasting plasma glucose concentration in patients with NIDDM. Diabetes 1994;43:1440-1444. [PubMed: 7958496]

50. Maggs DG, Buchanan TA, Burant CF, et al. Metabolic effects of troglitazone monotherapy in type 2 diabetes mellitus. A randomized, double-blind, placebo-controlled trial. Ann Intern Med 1998;128:176-185. [PubMed: 9454525]

51. Magnusson I, Rothman DL, Katz LD, et al. Increased rate of gluconeogenesis in type II diabetes mellitus. A 13C nuclear magnetic resonance study. J Clin Invest 1992;90:1323-1327. [PubMed: 1401068]

52. Hundal RS, Krssak M, Dufour S, et al. Mechanism by which metformin reduces glucose production in type 2 diabetes. Diabetes 2000;49:2063-2069. [PubMed: 11118008]

53. Landau BR, Wahren J, Chandramouli V, et al. Contributions of gluconeogenesis to glucose production in the fasted state. J Clin Invest 1996;98:378-385. [PubMed: 8755648]

54. Petersen KF, Krssak M, Navarro V, et al. Contributions of net hepatic glycogenolysis and gluconeogenesis to glucose production in cirrhosis. Am J Physiol 1999;276:E529-E535. [PubMed: 10070020]

55•. Mayerson AB, Hundal RS, Dufour S, et al. The effects of rosiglitazone on insulin sensitivity, lipolysis, and hepatic and skeletal muscle triglyceride content in patients with type 2 diabetes. Diabetes 2002;51:797-802. Demonstrated that thiazolidinedione therapy enhances insulin sensitivity in patients with type 2 diabetes by promoting increased insulin sensitivity in peripheral adipocytes. The results support the hypothesis that a redistribution of fat from insulinresponsive organs into peripheral adipocytes will improve insulin sensitivity. [PubMed: 11872682] 